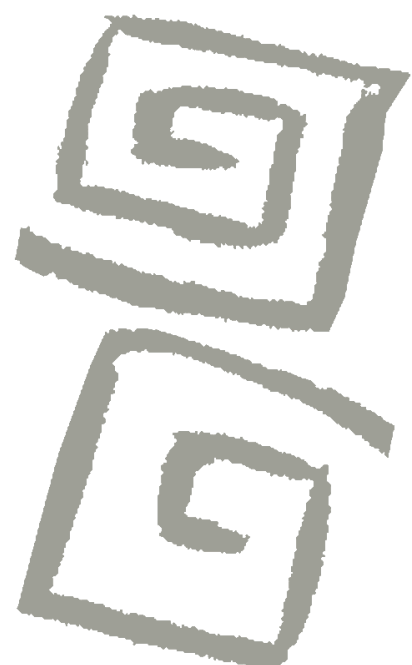

\title{
Sociogénesis de los dispositivos evangélicos de "rehabilitación" de usuarios de drogas en Argentina
}

\author{
Sociogenesis of evangelical devices for the \\ "rehabilitation" of drug users in Argentina
}

Joaquín Algranti', Mariela Mosqueira²

${ }^{1}$ Doctor en Ciencias Sociales. Investigador adjunto, Centro de Estudios e Investigaciones Laborales, Consejo Nacional de Investigaciones Científicas y Técnicas (CEIL-CONICET), Ciudad Autónoma de Buenos Aires, Argentina. $\square$ (iD)

${ }^{2}$ Doctora en Ciencias Sociales. Investigadora asistente, Centro de Estudios e Investigaciones Laborales, Consejo Nacional de Investigaciones Científicas y Técnicas (CEIL-CONICET). Ciudad Autónoma de Buenos Aires, Argentina. $\square$ iD
RESUMEN Para reconstruir el origen y desarrollo de los dispositivos neopentecostales dirigidos al tratamiento de adicciones en la Ciudad de Buenos Aires, Argentina, el artículo periodiza analíticamente los impulsos de "espiritualización terapéutica" de la conducta adictiva que emprenden los agentes evangélicos a partir de la década de 1970 y explora el modo en que el Estado y los grupos religiosos convergen en el Programa Recuperar Inclusión. Desde una metodología cualitativa y un enfoque sociológico entre 2015 y 2016, se realizaron catorce entrevistas a profesores, dirigentes y ex usuarios del programa, doce entrevistas a responsables estatales, observación participante en los cursos de formación y se conformó un corpus de documentos relativo a ambas instituciones. Los procesos emergentes de institucionalización política y religiosa se analizaron de acuerdo a tres dimensiones: a) el carisma y su par opuesto, la burocracia, b) al rol dinamizador de las segundas líneas y c) las estructuras de organización en red. Se concluye que existen encuentros y analogías entre las dinámicas de institucionalización evangélica y pública en el tratamiento de las adicciones.

PALABRAS CLAVES Religión; Conductas Adictivas; Tratamientos; Argentina.

ABSTRACT In order to reconstruct the origin and development of neo-Pentecostal devices for the treatment of addictions in the City of Buenos Aires, Argentina, the article analytically puts into historical context the "therapeutic spiritualization" of addictive behavior undertaken by evangelical agents since the 1970s and explores the way in which the State and religious groups converge in the Recover Inclusion Program. Based on qualitative methodology and a sociological approach and carried out in 2015 and 2016, the study includes fourteen interviews with teachers, leaders and former users of the program, twelve interviews with state officials, participant observation in training courses and a corpus made up of documents related to both institutions. The emergent processes of political and religious institutionalization were analyzed according to three dimensions: a) charisma and its opposite, bureaucracy, b) the dynamic role of the second lines of leadership and c) the networked organization structures. It is concluded that points of convergence and analogies exist in the dynamics of evangelical and public institutionalization in addiction treatment.

KEY WORDS Religion; Addictive Behaviors; Treatments; Argentina. 


\section{INTRODUCCIÓN}

Desde la década de 1990 a la actualidad, las imágenes religiosas del neopentecostalismo fueron las protagonistas de un doble movimiento de proyección, tanto estratégica como espontánea en la sociedad argentina. Por un lado, y siguiendo un criterio de alta visibilidad, los grupos evangélicos lograron establecerse en el espacio público ${ }^{(1,2,3)}$, consiguieron aumentar la escala y complejidad interna de sus organizaciones, accedieron geográfica y simbólicamente a las zonas de mayores ingresos $^{(4)}$, trabajaron con éxito dispar en la capacitación de líderes con influencia en la política $^{(5,6)}$ y los medios de comunicación, reforzaron una industria cultural específica, apoyada en las nuevas tecnologías ${ }^{(7,8,9,10)}$, crearon fundaciones para la asistencia a sectores desfavorecidos y consolidaron también un circuito de eventos y actividades orientadas, especialmente, a la construcción de un sujeto juvenil cristiano ${ }^{(11,12)}$. En fin, avanzaron -a fuerza de prueba y error, a través de biografías desorganizadas y sin un plan preestablecido pero con una dirección clara- hacia una institucionalización creciente de su presencia en la sociedad. De esta forma, incrementaron los fundamentos y las garantías, así como el carácter actual de su estilo de creencia.

Por otro lado, en esos mismos años, las acciones evangélicas estuvieron dirigidas a zonas menos visibles y deslumbrantes de la vida social. Las dos más importantes fueron la evangelización de las cárceles, esto es, la construcción de espacios propios, internos, sobre todo, en el Sistema Penitenciario Bonaerense $\mathrm{e}^{(13,14,15,16)}$, también en los institutos de menores $^{(17,18)}$. Y el trabajo con consumos problemáticos, es decir, el desarrollo de un método y una red especializada en el tratamiento de adicciones desde una perspectiva espiritual ${ }^{(19,20,21,22,23,24)}$. El medio carcelario y el consumo de drogas fueron dos áreas subterráneas de la proyección religiosa en la Argentina. En ambas se fueron definiendo progresivamente circuitos dinámicos, pero frágiles en el proceso de instituir modelos de intervención sobre dominios abandonados de la sociedad.
Recuperando en parte esta faceta subterránea de la praxis evangélica, el objetivo del artículo es explorar las terapéuticas neo-pentecostales dirigidas al tratamiento de adicciones en la Ciudad de Buenos Aires. Para comprender dichas iniciativas es preciso diferenciar analíticamente las dimensiones religiosas y estatales del fenómeno.

En este marco, estudiaremos en términos institucionales el territorio de intervenciones religiosas en consumos problemáticos y su peculiar relación con el Estado. Específicamente, reconstruimos: a) los procesos de espiritualización terapéutica de la conducta adictiva desplegados por agentes evangélicos desde la década de 1970, que se encumbran con la creación, en 2004, de la Red Nacional Cristiana de Rehabilitación, Capacitación y Prevención de Adicciones (Programa Vida) y el curso de Operador Socio-terapéutico Espiritual en Adicciones, en 2007; y b) el Programa Recuperar Inclusión coordinado conjuntamente por el Ministerio de Planificación y Obras Públicas y la Secretaría de Políticas Integrales sobre Drogas de la Nación Argentina (SEDRONAR). La articulación entre las redes evangélicas y las estatales presentan procesos análogos que exploraremos, atendiendo al papel del carisma y la burocracia, al dinamismo de las segundas líneas en cuestiones de liderazgo y a la concepción reticular del funcionamiento de las organizaciones.

Las expresiones "segundas líneas" o "cuadros medios", que utilizaremos frecuentemente a lo largo del artículo, remiten a una posición genérica, móvil y, en un sentido, inespecífica de autoridad que se caracteriza por participar activamente en la vida interna y las decisiones estructurales de las organizaciones, sin acceder a los círculos más selectos de conducción. La amplitud deliberada del término nos va a permitir establecer analogías entre, por ejemplo, el líder de un ministerio dentro de una megaiglesia y un funcionario de alto rango en la administración pública. Nos interesa reconocer el dinamismo y el carácter agonístico de estas posiciones, en tanto figuras profesionales que en el proceso de construcción de sus propias carreras dan inicio a formas emergentes de 
institucionalización que los impulsa tanto como los tensiona con las estructuras de procedencia.

\section{Antecedentes}

Los emprendimientos evangélicos orientados al tratamiento de usuario de drogas constituyen una temática escasamente explorada por las ciencias sociales. Considerando la producción académica local se registran los trabajos pioneros de Daniel Míguez ${ }^{(17,19)}$ quien indaga comparativamente programas estatales y pentecostales de rehabilitación de jóvenes adictos y delincuentes. Allí, el autor da cuenta de los mayores niveles de eficacia en la resocialización juvenil $-y$, por lo tanto, menores niveles de reincidencia delictiva o recaída adictiva- de los programas confesionales, centrándose en el estilo cognitivo, las sociabilidades y los contextos rituales propios del pentecostalismo como instancias fecundas de redefinición identitaria. En un estudio posterior, Míguez ${ }^{(20)}$ analiza narrativas de conversión de jóvenes insertos en programas pentecostales de rehabilitación de adicciones, poniendo de relieve la heterogeneidad de sentidos que adquiere la articulación entre la vida religiosa y el consumo de drogas, según la posición social y cultural del sujeto.

Por su parte, desde la antropología de la salud se registra el trabajo de Castilla y Lorenzo ${ }^{(22)}$, que explora las prácticas de cuidado y recuperación de usuarios de pasta base de cocaína y su vínculo con el pentecostalismo. Allí señalan que, dentro del contexto de extrema vulnerabilidad en el cual transcurren las vidas de los usuarios de pasta base de cocaína, el acercamiento al evangelio se configura como un eje privilegiado en las lógicas de "rescate", entendido como un conjunto de saberes, prácticas, operaciones y argumentos que regulan o detienen el consumo de drogas a partir de un vínculo social. En el mismo sentido, que los estudios de Míguez, las investigadoras destacan que el "rescate por el evangelio" posibilita la reestructuración subjetiva -mediante la resignificación biográfica a la luz de la cosmovisión religiosa- y abre canales de reinserción social a través de redes evangélicas, como el restablecimiento de lazos familiares y vecinales, el acceso a empleo, vivienda y capacitación. Asimismo, advierten que las terapéuticas evangélicas reafirman las lógicas de rescate informal al poner de manifiesto tanto la brecha entre usuarios y sistema de salud como la búsqueda de terapéuticas alternativas a las propuestas por los dispositivos de atención sanitaria.

Es preciso señalar que, a esta embrionaria trayectoria de estudios locales sobre iniciativas pentecostales de prevención y tratamiento del consumo de drogas, se suman los recientes trabajos de Jones y Cunial ${ }^{(23)}$ y de Güel$\operatorname{man}^{(24)}$. Los primeros abordan las dinámicas entre actores religiosos e instancias estatales en políticas públicas de drogas y, el segundo, presenta una descripción de comunidades terapéuticas evangélicas y católicas vinculadas a redes trasnacionales de atención.

Ampliando el foco al ámbito internacional, se observa una interesante producción académica en torno al eje religión y consumo problemático de drogas sintetizada en diversas revisiones bibliográficas ${ }^{(25,26,27)}$, en las que se enfatiza la existencia de una amplia variedad de estudios cuantitativos que asocian religiosidad con menor consumo de drogas o con mejores índices de recuperación en toxicómanos. También es posible reconocer un consenso en torno al modo en que la religiosidad y la espiritualidad resultan factores protectores ante el consumo de drogas, tanto en jóvenes como adultos, en diferentes contextos culturales. Dichas revisiones muestran, también, una carencia de estudios cualitativos que profundicen en la comprensión de las correlaciones cuantitativas señaladas y en los tratamientos religiosos/espirituales.

Es dable destacar, además, la trayectoria de investigaciones sobre usos de droga y espiritualidad gestada en Europa en la que sobresalen: a) el trabajo de Cantón Delgado ${ }^{(28)}$ quien aborda las iniciativas de tratamiento de "drogadictos" en el contexto del pentecostalismo gitano; b) el libro de Comas Arnau ${ }^{(29)}$ sobre centros residenciales de recuperación de adicciones de corte pentecostal; y c) el reciente estudio de Apud y Romaní(30) quienes 
reconstruyen redes y usos terapéutico-espirituales de la ayahuasca en Cataluña.

En Latinoamérica, por su parte, se ha desarrollado también una incipiente trayectoria de estudios sociales sobre toxicomanías y religión. Específicamente, registramos: a) el trabajo comparativo de Castrillón ${ }^{(31)}$ sobre laico-terapias y teo-terapias para drogodependientes en Colombia; b) la producción del equipo interdisciplinario del Colegio de la Frontera Norte (Universidad Autónoma de Baja California) quienes trabajan las ofertas terapéutico-religiosas de centros evangélicos de rehabilitación para fármaco-dependientes en Tijuana ${ }^{(32,33,34)}$; y c) las investigaciones sobre religión y consumo problemático de drogas desarrolladas en Brasil ${ }^{(35,36,37,38)}$.

Frente a este cuadro de situación en la literatura académica local, regional e internacional, la presente investigación se propone comprender los dispositivos evangélicos orientados al tratamiento de usuarios de drogas del Gran Buenos Aires (GBA). Esperamos contribuir a la profundización de los aspectos conocidos del objeto e incorporar nuevas claves de lectura relativas a la acción del carisma, los liderazgos intermedios y la estructura en red que gobierna a las organizaciones involucradas.

\section{ACERCA DE LA INVESTIGACIÓN}

Este artículo es resultado del Proyecto de Investigación Plurianual (PIP) 112-20150100375 (en curso) financiado y evaluado, ética y académicamente, por el Consejo Nacional de Investigaciones Científicas y Técnicas (CONICET) cuyo objetivo general es reconstruir las formas sociales de los objetos y programas terapéuticos orientados al tratamiento de usuarios de drogas por parte de organizaciones evangélicas del Gran Buenos Aires. Es propósito de esta investigación enriquecer la trayectoria de estudios sociales sobre salud y religión ${ }^{(39,40,41,42,43,44,45,46,47,48,49,50)}$ y sobre religión y adicciones ${ }^{(31,32,33,34,35,36,37,38,51,52)}$ desarrolladas en el ámbito regional en las últimas décadas.
En el marco analítico de la sociología de la religión y desde un enfoque cualitativo, realizamos trabajo de campo intensivo en el período 2015-2016, tomando como puntos de observación prioritarios la Red Nacional Evangélica de Rehabilitación, Capacitación y Prevención de Adicciones (Programa Vida) y al Programa Recuperar Inclusión.

Específicamente, dentro del Programa Vida, realizamos observación participante del curso de formación de Operadores SocioTerapeutas Espirituales en Adicciones (OSTE) que dicta el programa de modo regular desde 2007. La modalidad de cursada fue semanal, en clases de tres horas emplazadas en cuatro iglesias evangélicas de la zona norte y noroeste del Gran Buenos Aires. Todos los registros etnográficos fueron recopilados en diarios de campo.

La estadía en los cursos OSTE y los vinculos gestados con los dirigentes del Programa Vida, habilitaron la realización de entrevistas en profundidad a catorce profesores, dirigentes y ex usuarios del Programa Vida. Los entrevistados, seleccionados a partir de criterios de contigüidad social, nos permitieron el acceso a documentos de la Red y a sus archivos personales, que constaban de un volumen importante de material empírico (fotos, reglamentos, folletería, literatura).

Dentro de la órbita del Programa Recuperar Inclusión, por su parte, durante 2016, se relevaron documentos relativos a la política pública y se efectuaron un total de doce entrevistas abiertas, extensas y recurrentes a agentes estatales involucrados, quienes fueron seleccionados siguiendo el proceso de muestreo de bola de nieve. Las entrevistas fueron realizadas en las oficinas del Programa Vida y del Centro de Estudios e Investigaciones Laborales, Consejo Nacional de Investigaciones Científicas y Técnicas (CEILCONICET). Todas fueron grabadas $y$, posteriormente, transcriptas.

Paralelamente, y a los fines de reconstruir históricamente el objeto de estudio, se realizó un relevamiento de notas, sobre el consumo de drogas, publicadas por el periódico cristiano El Puente, durante el período 1985-2010. 
Es dable destacar que en cada una de las instancias del trabajo de campo se obtuvo el consentimiento informado de las personas involucradas en la investigación y se les garantizó confidencialidad y anonimato.

La sistematización y el procesamiento de los datos relevados supusieron el diseño de una estrategia mixta de abordaje mediante el programa de análisis cualitativo Atlas$\mathrm{Ti}$, el de análisis reticular UCINET 6 y NetDraw. Esta estrategia combinada de análisis permitió: a) la reconstrucción histórica del Programa Vida, b) el registro de las distintas articulaciones e interacciones del Programa Vida con agentes político-estatales durante las presidencias de Néstor Kirchner y Cristina Fernández y c) los sentidos sedimentados en torno a las terapéuticas y dispositivos evangélicos de atención a usuarios de drogas.

\section{RESULTADOS}

\section{"Espiritualización terapéutica" de la conducta adictiva: transformaciones en los dispositivos evangélicos de rehabilitación}

La sociogénesis de los dispositivos evangélicos en salud reconoce tres impulsos de "espiritualización terapéutica" de la conducta adictiva. El primero, se inicia en 1973 con la creación del "Grupo Andrés" en la localidad de Villa Adelina (zona norte del Gran Buenos Aires) por iniciativa del Pastor Carlos Novelli. El segundo impulso, comienza al despuntar la década de 1980 y supone la emergencia de una constelación de emprendimientos locales de evangelización y asistencia a "drogadictos" (ministerios radiales, musicales, grupos de autoayuda). El concepto de "ministerio" dentro del mundo evangélico refiere a organizaciones para-eclesiásticas o a departamentos dentro de las iglesias que se enfocan en un área de servicio específica. Como mostraremos, estas experiencias no fueron parte de una estrategia institucional diseñada desde las iglesias, sino que fueron dinamizadas por sectores de la propia feligresía pentecostal en crecimiento. Finalmente, el tercer impulso surge en la década de 1990, fruto de la transnacionalización de ministerios evangélicos de "rehabilitación de adictos" procedentes de Europa y EEUU.

En los próximos subapartados profundizaremos en cada impulso, mostrando sus lógicas de emergencia e institucionalización en las que se destacan tres aspectos centrales: a) el carisma de los emprendedores y la progresiva burocratización/estandarización de los ministerios, b) el dinamismo de líderes evangélicos de rango medio o periféricos en la conformación y sostenimiento de estas iniciativas, y c) la lógica reticular que adoptan los proyectos enlazándose transversalmente tanto con las iglesias como con la sociedad civil y el Estado.

\section{La experiencia del Pastor Novelli}

La experiencia de Carlos Novelli se imbrica con las dinámicas que adoptó el "problema de la droga" durante 1960 y 1970 cuyo foco fueron los circuitos juveniles de clase media vinculados a la cultura rock y las vanguardias artístico-intelectuales ${ }^{(53,54)}$. NoveIli, justamente, era un joven perteneciente a esos círculos sociales, era usuario y tratante de drogas al menudeo de la zona norte del Gran Buenos Aires. En el año 1972, en uno de sus viajes a EEUU para abastecerse de la cocaína que luego vendería a su círculo de amigos y conocidos, Novelli tuvo un "encuentro con Dios" y abandonó totalmente el consumo y el pequeño tráfico ${ }^{(55,56)}$. Aunque no se sabe a ciencia cierta con qué experiencia terapéutica tuvo contacto, podemos intuir que lo hizo con algún emprendimiento evangélico influenciado por el movimiento antiprofesional y antiinstitucional inspirado en Alcohólicos Anónimos que, por aquel entonces, promovía estrategias de rehabilitación para drogodependientes con impronta jerárquica basadas en la autogestión y la autoayuda (por ejemplo, los programas Daytop y Synanon) $)^{(56,57)}$.

A su regreso -en 1973- Novelli fundó el "Grupo Andrés" en los fondos de su casa en Villa Adelina. Allí comenzó a dar contención a sus antiguos clientes/amigos adictos. 
Estos encuentros esporádicos consistían en momentos de lectura bíblica e intercambio de experiencias con la finalidad de sostener la abstinencia.

El emprendimiento de Novelli fue autónomo durante los primeros tiempos y, recién en 1976, logró articularse con la Iglesia Evangélica Bautista de La Lucila. Primero se acercó como fiel y, más tarde, Novelli fue autorizado a trasladar sus grupos de autoayuda a las instalaciones del templo. Según nuestros registros, ese fue el único vínculo estable que Novelli mantuvo con la comunidad evangélica. Es más, aunque se lo referencia como "pastor", él no tuvo feligresía a cargo, ni tomó cursos en seminarios bíblicos. Solo fue nombrado "obrero" por la Iglesia Evangélica Bautista de La Lucila, para oficiar sus reuniones con ex adictos y evangelizar.

Hacia 1982, Novelli abrió el Centro de Rehabilitación Cristiano de Adicciones "Programa Andrés", en la localidad de Diego Gaynor, constituyéndose en la primera comunidad terapéutica residencial de la Argentina. La dinámica de la "granja" o "comunidad de vida" ${ }^{\prime \prime 7,58)}$ consistía en el mantenimiento voluntario/comunitario de la abstinencia bajo fuertes convicciones religiosas y, económicamente, se sostenían con la ayuda de los familiares de los residentes, con ofrendas procedentes de organizaciones religiosas $y$, también, con la producción de objetos artesanales y/o comestibles.

Con el correr de la década de 1980, los emprendimientos de Novelli fueron cobrando visibilidad entramándose con iniciativas similares laicas y religiosas emergentes desde la sociedad civil como "Fundación Viaje de Vuelta" o "Fundación Convivir" y con referentes claves de la psiquiatría comunitaria y el psicoanálisis como Wilbur Grimson $^{(56)}$. Los vínculos generados por su condición de "pionero", por su personalidad frondosamente referida como "carismática" y por su procedencia social (vinculada a sectores medios y altos) hicieron del "Pastor Novelli" una figura central de la nueva escena terapéutica no-gubernamental en adicciones. Posición que lo llevó a fundar, en el año 1986, la Federación de Organizaciones no
Gubernamentales de la Argentina para la Prevención y el Tratamiento del Abuso de Drogas (FONGA) y, en 1987, a viajar en calidad de presidente de la institución a la reunión anual de la Junta Internacional de Estupefacientes de las Naciones Unidas ${ }^{(56)}$.

La inserción en redes internacionales lo vinculó estrechamente con la Embajada de EEUU y con el Progetto Uomo de Italia. Los lazos con la primera, devinieron en la producción de material editorial precursor en el país en materia de prevención de adicciones para colegios. Específicamente, en 1988 y 1989, el Programa Andrés editó la colección "Los niños y las drogas" integrada por las traducciones al español del libro Peligro Marihuana y de la saga La verdadera historia de Mary Juana, de la periodista estadounidense Peggy Mann, condecorada por el Congreso de su país natal como la "autora más notable de la Nación sobre prevención del abuso de drogas"(59). Por su parte, el contacto con Progetto Uomo (método de rehabilitación de drogodependientes creado en 1979 por el sacerdote católico Mario Picchi) dinamizó la primera formación de operadores socioterapéuticos del país impulsada desde Naciones Unidas. Estas capacitaciones fueron determinantes tanto para la difusión del modelo italiano en Argentina como para la profesionalización de los referentes de las "comunidades de vida" pioneras ${ }^{(56,57,58)}$.

La estructuración de la escena terapéutica no-gubernamental pone de manifiesto la institucionalización y profesionalización que progresivamente transitaron los emprendimientos laicos y religiosos orientados al tratamiento de "drogadictos" emergentes desde la sociedad civil, proceso que termina encumbrándose con la creación de la SEDRONAR, en 1989, y la incorporación de muchas de estas iniciativas dentro de la órbita estatal. Finalmente, con la muerte de Novelli, en 1994 FONGA queda bajo la dirección de Wilbur Gimson y "Programa Andrés" inicia un derrotero de descristianización, aunque sin perder la dimensión espiritual en sus tratamientos, dado que actualmente se realizan actividades vinculadas al yoga y la meditación en articulación con la organización el "Arte de Vivir" de Ravi Shankar. 


\section{Emprendimientos pentecostales locales y transnacionales}

El segundo impulso evangélico de "espiritualización terapéutica" de la conducta adictiva, se inicia con el regreso democrático y coincide con los procesos de renovación, pentecostalización y apertura cultural que estaban desplegándose en el campo evangélico. Esta reconfiguración incluye la emergencia de líderes carismáticos con estilos propios, formas de evangelización innovadoras, nuevos énfasis teológicos y flexibles estructuras institucionales. Este conjunto amplio de transformaciones, es acompañado del ingreso de una gran cantidad de conversos que pasan del $2,6 \%$ en 1960 al $9 \%$ de la población argentina a fines de la década de 1990, manteniéndose en esas cifras hasta nuestros días ${ }^{(1,4,11)}$.

Durante esa etapa de crecimiento, emerge una constelación de emprendimientos locales de evangelización y asistencia religioso-terapéutica para jóvenes consumidores de drogas, que abarcaron desde proyectos culturales como programas de radio, conjuntos de rock y festivales hasta alternativas de tratamiento como grupos de autoayuda o centros de rehabilitación.

Dentro de las iniciativas evangelísticas orientadas a personas "adictas" se destaca el ministerio "Dios te bendiga", impulsado por un joven "ex rockero y ex adicto" converso al evangelio, quien a mediados de la década de 1980 decidió salir a predicar en boliches de la zona oeste del Gran Buenos Aires. Su ministerio contó con un programa radial y desarrolló varios festivales titulados "Rock y $\mathrm{Fe}^{\prime \prime(60)}$. Esta mentada asociación entre drogas y rock inspiró también la conformación de una gran cantidad de bandas musicales que buscaron "rescatar" a jóvenes usuarios de drogas mediante la fe, entramándose en la escena underground rockera secular. Entre los conjuntos más dinámicos del circuito se encuentra el ministerio "Boanerges", que inició sus actividades en 1988 vinculándose a la cultura heavy metal. También, podemos mencionar a "Tommy y la banda de Jesús" que, en sus líricas, por ejemplo, interpelaba a los toxicómanos en estos términos:
Basta de porros, basta de coca, basta de pastas, basta de mal. Yo solo quiero seguir a Cristo que Él una nueva vida me dará [...] Yo no quiero convencerte solamente con palabras, yo quiero que me mires y digas qué ves en mí. Estuve encerrado en el mundo de las drogas, pero el Señor me salvó, me rescató. (Fragmento del tema "Basta de mal")

Junto a estas actividades artístico-evangelísticas se fueron desarrollando iniciativas pentecostales dirigidas al tratamiento de "drogodependientes" con la modalidad de grupos de ayuda mutua y/o "comunidad de vida". Una de las pioneras fue el "Centro de Rehabilitación para Drogadictos Arca de Noé", que surgió durante 1982 en un barrio marginal del Partido de Escobar por iniciativa del naciente ministerio "Ondas de Amor y Paz" del pastor Héctor Giménez. Como señala Spadafora(61) la vinculación de Giménez con la "recuperación" de "adictos" se enraizó con su propia historia personal que anuda un pasado de adicciones, delincuencia y pobreza.

Esta característica, sin embargo, no es exclusiva de Giménez, sino que se extendió en la gran mayoría de los ministerios pentecostales emergentes durante 1980 y 1990, los cuales fueron generalmente impulsados por personas de bajos recursos, ex adictas $y$, en algunos casos, con pasado delictivo, conversas al evangelio. El origen social de los emprendedores pentecostales, entonces, pone de manifiesto que estos proyectos religioso-terapéuticos se imbricaron en un espacio social distinto al del "Programa Andrés" articulado -como vimos- a sectores medios. De manera clara, estos impulsos de rehabilitación fueron reverberando entre sectores empobrecidos y marginados, en el mismo momento que el pentecostalismo y que el "problema de la droga" se territorializaban allíi $(19,20,21,62,63,64)$.

La experiencia de Giménez también marca una diferencia ya que los ministerios pentecostales en adicciones no fueron dinamizados por "pastores", sino por líderes de mediano rango o por miembros periféricos 
de la feligresía. Esta condición revela el carácter autónomo y autogestivo de los emprendimientos $y$, también, las tensiones que fueron suscitándose con los propios pastores de las iglesias.

A los pastores nunca les gustó que la iglesia se les llenara de drogadictos [...] En la iglesia nos daban un lugar muy reducido para armar los grupos, parecíamos un gueto dentro de otro gueto [...] Los pastores no sabían nada de drogas por eso no podían entender nuestro ministerio. (Entrevista a A, ex dirigente y miembro del grupo fundador de Programa Vida)

Este tipo de expresiones aparecen continuamente en las entrevistas, incluso los miembros de la banda Boanerges lo expresaban al periodismo confesional en 1998:

\begin{abstract}
Hacemos lo que Jesús hacía. Estamos con aquellos que la iglesia no puede llegar. No hacemos acepción de personas. Jesús estaba con gente que era mal vista. Creo que hay que cambiar las estructuras de la iglesia para que puedan recibir gente así. ${ }^{(65)}$
\end{abstract}

Claramente, estos impulsos no fueron parte de un diseño institucional de abordaje de las adicciones por parte de las iglesias evangélicas en su conjunto. Por el contrario, se trató de emprendimientos independientes dinamizados por líderes de mediano rango en las iglesias o por miembros de la vasta feligresía general. Desde una sociología de las figuraciones $^{(66)}$, podemos decir que es el encadenamiento de voluntades y acciones dispersas en sus orígenes, pero que al pertenecer a entramados de relaciones análogos desembocan en un proceso común. Es este proceso en marcha al que se dota retroactivamente de una idea de planificación, dirección y conducción, de la cual carecía en sus inicios.

Paralelamente a estas iniciativas autónomas, comenzaron a instalarse en el país ministerios pentecostales de rehabilitación de adictos de corte transnacional. Entre los más renombrados podemos citar a "Remar", "Reto a la vida" y "Desafío Juvenil". Los dos primeros, son originarios de España y surgen al despuntar 1980 como consecuencia de impulsos misioneros autónomos procedentes de los EEUU ${ }^{(29)}$. Luego de afianzarse en distintas regiones de España, inician un proceso de internacionalización que los lleva a desembarcar en la Argentina en 1992. Por su parte, Desafío Juvenil es un ministerio procedente de los EEUU, iniciado en 1958 por el predicador David Wilkerson, que arriba al país también a comienzos de 1990 en articulación con la denominación pentecostal "Unión de las Asambleas de Dios".

Estos ministerios transnacionales implican un tercer impulso de "espiritualización terapéutica" de la conducta adictiva porque su llegada supuso la implantación de comunidades terapéuticas pentecostales residenciales que, en vínculo con los emprendimientos autónomos ambulatorios antes descriptos, configuran una escena terapéutica pentecostal de dispositivos de "rehabilitación" de adictos con gran presencia en sectores de vulnerabilidad social.

\section{Programa Vida y Curso OSTE}

La escena terapéutica pentecostal de recuperación de "drogodependientes" dinamizada por los emprendimientos autónomos y transnacionales irá progresivamente interconectándose y, con el transcurrir de la década de 1990 se entramará de modo transversal a las iglesias evangélicas, operando como espacios de consulta, capacitación y derivación de casos.

La experiencia acumulada y los vínculos generados entre ministerios propician la constitución de dos instancias de institucionalización y profesionalización de estos impulsos: la creación en 2003 de la Red Nacional Evangélica de Rehabilitación, Capacitación y Prevención de Adicciones "Programa Vida" y la puesta en marcha del "Curso de Operador Socio-Terapéutico Espiritual en Adicciones" (OSTE), en 2007.

"Programa Vida" surge por iniciativa de cuatro ministerios "Viviré", "Programa Josué", 
"Bernabé" y "Formación Evangélica en Salud Mental" (FESAM). El primero, se orientaba a la capacitación de iglesias para la formación de grupos de autoayuda y estaba a cargo de un ex adicto converso que había servido por diez años como líder principal en los dispositivos de internación de Desafío Juvenil y debido a su expertise y contactos acumulados era uno de los referentes centrales de la escena terapéutico-pentecostal en adicciones. El Programa Josué es un centro de rehabilitación de día y se vincula a la mega-iglesia "Catedral de la Fe" de Parque Chacabuco. Fundado en 1995 por un adicto recuperado en Desafío Juvenil, es hasta hoy el único dispositivo cristiano semiambulatorio. Los ministerios Bernabé y FESAM, por su parte, se conectaban con la "Iglesia de la Puerta Abierta" de Villa Devoto. Bernabé, era un grupo de atención primaria en adicciones que sesionaba en dicha iglesia desde 1992 liderado, también, por un ex adicto converso. Finalmente, FESAM es una asociación civil evangélica dedicada a la formación y asistencia en salud mental cuya directora en esa época era una médica psiquiatra evangélica miembro de la congregación de la Puerta Abierta. En su acta fundacional de 2003, Programa Vida establece:

Hace años que muchos grupos grandes y pequeños en diferentes congregaciones vienen desarrollando aisladamente esta tarea. Y la realidad que surge de las estadísticas es que el índice de la recuperación de estos grupos es largamente mayor que el de instituciones seculares y oficiales. ¡Porque Jesús puede! Creemos que ha llegado el tiempo de capitalizar las experiencias. Queremos mostrar ese poder como cuando Elías encendió la pira. No por jactancia sino pensando en los oprimidos por las drogas.

Estos cuatro ministerios fundacionales, se abocaron a la tarea de interconectar a los emprendimientos de la Ciudad Autónoma de Buenos Aires y, en su lanzamiento, contaron con la adhesión de 19 ministerios dedicados a la recuperación de adictos. Actualmente, poseen una nómina de 150 entidades distribuidas en todo el país.

Programa Vida nuclea a la gran mayoría de dispositivos evangélicos en adicciones existentes de la Argentina en sus tres modalidades: grupos de ayuda mutua, hospital de día y comunidades terapéuticas residenciales. En términos prácticos, funciona de modo similar a la SEDRONAR, pero dentro de la estructura evangélica. Es decir, opera como un "derivador", que recibe casos desde las iglesias o desde la sociedad civil y luego de una serie de entrevistas con psicólogos y operadores socioterapéuticos cristianos, se decide qué tipo de tratamiento requiere la persona y, de acuerdo a su ubicación geográfica, se lo conecta al ministerio local y se hace un seguimiento.

Otra de las funciones centrales de Programa Vida es "capacitar" a las iglesias en la temática adictiva y, para ello, durante estos años se abocó a la tarea de sistematizar la experiencia de los múltiples emprendimientos autónomos y en función de esa labor se sintetizó la expertise acumulada en lo que se dio en Ilamar el Curso de Operador SocioTerapéutico Espiritual en Adicciones (Curso OSTE).

Desde su creación, en 2007, se dictaron 50 cursos en todo el país, que capacitaron cerca de 1.500 operadores. El curso es arancelado -los estudiantes pagan una módica cuota mensual- y cuenta con un total de 10 materias que se cursan en modo intensivo o regular. En cuanto a las temáticas que se abordan, podemos mencionar: prevención integral de adicciones, nociones básicas de psicología y psiquiatría, conceptos químicos y farmacéuticos generales, el rol del operador y las dinámicas de grupo, teología y técnicas espirituales aplicadas al tratamiento de adicciones.

El cuerpo docente del Curso OSTE está conformado por la dirigencia de Programa Vida que, en lo sustantivo, se compone de los fundadores del Programa o personas vinculadas a ellos; todos con una vasta expertise práctica y, también, profesional en la materia, como psicólogos, psiquiatras, operadores socioterapéuticos, trabajadores sociales y docentes. 
Programa Vida y el Curso OSTE suponen una instancia de institucionalización, profesionalización y, por ende, homogeneización de los "tratamientos" evangélicos de rehabilitación de adictos en la Argentina. Esos movimientos, sin embargo, no implican un proceso de "descristianización" o "secularización" de las propuestas terapéuticas sino, por el contrario, se observa una estabilización del trabajo de síntesis discursiva y práctica que vienen realizando los ministerios evangélicos entre abordajes religiosos y sociosanitarios de la conducta adictiva.

\section{La interpretación del Estado}

\section{"El Programa es todo": sobre las políticas tradicionales, transversales y trascendentes}

Como analizamos en el apartado anterior, los diferentes impulsos de "espiritualización terapéutica" de las pautas adictivas fueron sostenidos por un modelo emergente de institucionalización, en el que se destacan tres rasgos dominantes:

a. el carisma de los iniciadores, es decir, la construcción extraordinaria de las cualidades, las visiones y aptitudes de aquellas personas que llevan adelante proyectos propios; la cual es habilitada y reforzada simbólicamente por el sistema de creencias evangélico, sobre todo, de aquellos que tienen éxito en nuevas áreas de intervención religiosa;

b. la posición que ocupan estos iniciadores en las organizaciones existentes: no es el pastor principal sino los lideres secundarios -a los que podríamos describir como cuadros medios o segundas líneas en un lenguaje sociológico- los que dinamizan las instituciones bajo la lógica del cisma, la innovación y la posibilidad de especializarse en realidades no abordadas por las iglesias;

c. el esquema reticular que adoptan los Programas al proponer un vínculo de transversalidad con distintos agentes del medio evangélico, pero también del Estado en todas sus escalas. Las redes se estabilizan en zonas de alta circulación de individuos, recursos y saberes -los denominados núcleos duros- y en zonas periféricas marcadas por la velocidad y el dinamismo de las sociabilidades débiles.

En una palabra, frente a la pregunta por la sociogénesis de las instituciones evangélicas que trabajan con adicciones es central tener en cuenta la construcción carismática de los fundadores, su posición subordinada en las estructuras de origen y el modelo en red que adoptan sus programas.

Ahora bien, ¿qué ocurre cuando estas organizaciones reticulares entran en contacto y construyen articulaciones duraderas o efímeras con el Estado? Nos proponemos retomar analíticamente las dimensiones señaladas relativas al carisma, los cuadros medios y las estructuras en red-para explorar el origen de la primera política pública que adaptó elementos y saberes religiosos a la intervención estatal sobre consumos problemáticos: nos referimos al Programa Recuperar Inclusión, iniciado a fines del 2014.

Podemos comenzar retomando dos definiciones taxativas que realizaba durante el trabajo de campo uno de nuestros principales informantes y creador de dicho programa. Este ex funcionario del antiguo Ministerio de Planificación y Obras Públicas, un hombre versado en el funcionamiento de los mecanismos estatales, en el diseño y gestión de políticas concretas de abordaje territorial, enfatizaba, como primera definición de su tarea, la importancia central que tienen los programas para el Estado. A nivel de la administración pública, afirmaba, "el Programa es todo". Por debajo del plan estratégico que desarrolla el gobierno para sus diferentes carteras, pero por encima de las acciones efectivas en el territorio, se encuentra el programa, que representa una propuesta de intervención sobre problemáticas específicas de la sociedad con base en un abordaje integral. Este contempla una fundamentación política, el marco normativo que lo avala, acciones preliminares y complementarias, objetivos generales y específicos, modelos de gestión, financiamiento y distribución de 
responsabilidades, roles y tareas entre todos los agentes involucrados. En más de un sentido, el Estado funciona, piensa y trabaja a través de los programas que llevan adelante los ministerios. Esta dimensión teórico-práctica del aparato estatal es clave en la introducción de innovaciones en la forma de enfocar y construir una temática, como es el caso de los consumos problemáticos y la incorporación de una metodología cercana a las técnicas evangélicas y católicas de tratamiento.

La segunda definición que introdujo nuestro informante fue una caracterización nativa de los tipos de políticas públicas que despliegan los gobiernos, que diferencian los esquemas "tradicionales", los "transversales" y los "trascendentes". Cada uno de ellos supone distintas lógicas de relación del Estado con la sociedad y sus áreas de intervención.

En el esquema "tradicional" las políticas se encuentran fuertemente centralizadas -a nivel del diseño, el financiamiento, la ejecución y el control- en grandes instituciones que concentran la totalidad de las actividades públicas sobre problemáticas y poblaciones específicas. Este modelo de acción estatal encuentra una de sus expresiones paradigmáticas -aunque no la única- en las primeras experiencias de gestión del peronismo entre 1946 y 1955.

Por el contrario, las políticas "transversales" plantean, para nuestro entrevistado, el desafío de la articulación eficiente y flexible entre distintos ministerios que deben coordinar de manera conjunta sus actividades al participar de la misma política pública. Es una articulación intraestatal, pero también con las diversas escalas del territorio, es decir, con los gobiernos provinciales y municipales. Se trata de una política que busca descentralizar las áreas públicas para pensar las intervenciones en términos de redes.

Por último, el tercer modelo es el "trascendente" en el que el Estado se propone ejecutar políticas a través de las organizaciones de la sociedad civil que cuentan con conocimientos, técnicas de trabajo y anclaje territorial en temas específicos. En este caso la política:

\begin{abstract}
...trasciende la raya del Estado, no es que te asisto únicamente, sino que yo quiero ejecutar cosas a través tuyo para que vos después continúes; entonces, salís del centralismo del Estado, trascendés esa línea, ese límite que había entre el Estado y la sociedad y te metés en una sinergia adentro de la sociedad. (Entrevista a L, ex funcionario del antiguo Ministerio de Planificación y Obras Públicas)
\end{abstract}

En una palabra, en las acciones trascendentes las políticas públicas integran en su diseño la necesidad de impulsar a determinados actores que funcionan por fuera de las estructuras gubernamentales. El "modelo trascendente" es la expresión nativa, religiosamente informada, que utiliza nuestro entrevistado para referirse a lo que en la administración pública contemporánea se conoce como "gobernanza" o "gobierno relacional", esto es, la concepción estratégica de la gestión estatal con base en la incorporación de las iniciativas de la sociedad civil en el desarrollo y la implementación de políticas de Estado. La especificidad de la idea de "trascendencia" remite, en nuestro caso, a una espiritualización refigurada de la gobernanza a través del protagonismo en ascenso de los dispositivos religiosos -tanto católicos como evangélicos- en el tratamiento de las adicciones.

Los últimos dos modelos son compatibles entre sí. Una política puede incluir principios transversales y trascedentes en el abordaje de una problemática particular. La importancia del Programa -en tanto mecanismo de pensamiento-acción del Estadoy las innovaciones en materia de políticas públicas contribuyeron fuertemente al desarrollo de una nueva forma de abordar los consumos problemáticos. La novedad radica en la incorporación de la expertise y la metodología de los grupos religiosos a los dispositivos públicos que trabajan en el tratamiento y prevención de adicciones. 


\section{Programa Recuperar Inclusión: analogías políticas y religiosas de la función pública}

En correspondencia con las iglesias, los procesos estatales de institucionalización ponen en juego elementos relativos a las formas del carisma -y su par opuesto, la burocracia-, al dinamismo de las segundas líneas y a la concepción reticular de las organizaciones. El Programa Recuperar Inclusión es un ejemplo de cómo se articulan estas tres dimensiones de análisis. Podemos introducirlo brevemente como un plan integral en el abordaje de consumos problemáticos a través de la creación de Casas Educativas Terapéuticas (CET), Centros Preventivos Locales de las Adicciones (CePLA) y el otorgamiento de subsidios a organizaciones de la sociedad civil que abordan la temática.

La primera dimensión, relacionada con la lógica del carisma y la burocracia, describe un aspecto central del Programa. Este es elaborado, primero, en toda su complejidad normativa, financiera y estratégica, por funcionarios del Estado que hacen confluir la imaginación política y burocrática en un plan de acción detallado, un documento de trabajo. Las tareas preliminares de la administración pública anteceden al lanzamiento masivo del Programa a cargo de la figura central de la expresidenta Cristina Fernández de Kirchner y las circunstancias extraordinarias que rodean su performance política. El anuncio se realiza en el marco de un evento multitudinario en el que se reactualizan y escenifican los sentidos fuertes, las bases, mitos y los símbolos del partido político que preside (Frente para la Victoria). A diferencia de las instituciones evangélicas -tal es el caso de Programa Vida- en las que el carisma de los iniciadores es construido en la práctica misma, es decir, en el ejercicio efectivo de su tarea, previo a una burocratización mínima de sus actividades, en este caso el Estado diseña hasta el detalle el plan de acción a seguir y luego adhiere, consolida, el impulso carismático de sus máximos referentes.

La segunda dimensión nos lleva a considerar la posición clave que ocupan las segundas líneas o cuadros medios en la dinamización de las instituciones. El Programa Recuperar Inclusión fue originalmente ideado y conducido en cada una de sus etapas de desarrollo por un funcionario de alto rango, próximo al ministro de Planificación y Obras Públicas. Sus intereses, sus preocupaciones biográficas, sus redes internas y apuestas profesionales en la administración pública son las que impulsan la elaboración colectiva de un programa de abordaje integral de la salud focalizado en las problemáticas de consumo.

A su vez, este Plan de Acción requiere de los tiempos políticos para su lanzamiento que dictaminan las instituciones involucradas (el Ministerio de Planificación, el Ministerio de Salud y la SEDRONAR) con la Presidencia de la Nación a la cabeza. En este sentido, cabe destacar el nombramiento, en 2013, de un cura católico, creador de la Fundación Valdocco, especializada en el tratamiento de adicciones, delante de una institución pública: la SEDRONAR. La designación de un sacerdote con experiencia de trabajo en la problemática obedece, en parte, a un cambio de perspectiva que introduce la Ley 26.657 de Salud Mental (sancionada en el 2010 y reglamentada en el 2013) y la Ley 26.934 Plan Integral para el Abordaje de los Consumos Problemáticos (Plan IACOP) de 2014. Ambas leyes contribuyen a redefinir el marco normativo y las modalidades de abordaje en salud bajo el paradigma de los derechos humanos. El secretario ejerció su cargo hasta el 2015. Su gestión coincide con el lanzamiento y aplicación del Programa Recuperar Inclusión y con la incorporación del punto de vista religioso en el diseño de políticas públicas dirigidas al tratamiento y prevención de adicciones.

Nos interesa señalar el papel activo de este funcionario de segunda línea que dinamiza transversalmente uno de los principales ministerios del Estado. Durante este proceso introduce innovaciones y articulaciones inesperadas con organizaciones evangélicas de las cuales toma, como vamos a ver, una metodología de trabajo, pero traducida al lenguaje y a las formas de las políticas públicas. Las innovaciones se encuentran vinculadas, 
en parte, a su propia experiencia creyente que lo sensibiliza sobre ciertos temas y modelos de tratamiento y a un contexto político que habilita esta lectura. Podemos insistir nuevamente en cierta analogía con los procesos evangélicos de institucionalización: los líderes y no tanto los pastores, las segundas y no las primeras líneas en clave política, son los que introducen cambios emergentes en las estructuras organizativas.

La tercera dimensión de análisis que nos gustaría recuperar consiste en la estructura en red que presenta el Programa Recuperar Inclusión. El diseño reticular que gobierna la política pública aparece en dos niveles complementarios, en los que la concepción "transversal" y "trascendente" del Estado en su relación con la sociedad se articulan entre sí. Por un lado, el Programa involucra las actividades conjuntas de distintos ministerios en las casas terapéuticas y los centros preventivos. Esto supone un trabajo de coordinación entre diferentes carteras del Estado: el Ministerio de Planificación realiza las inversiones en infraestructura, aporta las herramientas tecnológicas y conforma los denominados equipos de vitalizadores, sobre los cuales vamos a extendernos más adelante; la SEDRONAR identifica las zonas de vulnerabilidad, define los perfiles laborales y las metodologías de trabajo de los equipos interdisciplinarios, gestiona el abordaje territorial de los profesionales, supervisa su desempeño, otorga los recursos básicos de funcionamiento y brinda subsidios a organizaciones de la sociedad civil que trabajan en consumos problemáticos. Finalmente, el Ministerio de Salud es responsable de la selección del personal en atención terapéutica y la gestión de plazas en hospitales generales para los casos que requieren de una internación. Otros ministerios como el de Educación, Trabajo, Justicia y Desarrollo Social también participan, pero en menor medida por medio de cursos, capacitaciones y asesoramientos.

Por otro lado, el Programa despliega no solo una política intraestatal, sino también una estructura de trabajo en red con actores no gubernamentales que abordan la prevención y tratamiento de adicciones. Aquí es donde, siguiendo al sistema de clasificación de nuestro informante, las políticas "transversales" se vuelven "trascendentes" porque el Estado adopta el rol de mecenas o, al menos, de impulsor de las organizaciones que trabajan efectivamente en el territorio. Esto es lo que ocurrió, por ejemplo, con el Programa Vida en tanto actor paradigmático del trabajo evangélico sobre consumos problemáticos. El marco interpretativo del Programa Recuperar Inclusión otorgó un cierto grado de reconocimiento y, por lo tanto, de legitimidad pública al accionar de los grupos religiosos, incluyendo al neo-pentecostalismo en un rol protagónico. Se otorgaron subsidios y financiamientos para la construcción de casas terapéuticas en zonas aledañas a las iglesias, como es el caso de Catedral de la Fe de Parque Chacabuco. De esta manera, el modelo reticular de los programas estatales se articula con las redes evangélicas, produciendo así una zona de encuentro entre dos lógicas de anclaje en el territorio que carecían hasta entonces de un encuadre político que institucionalizara esta relación. Para el gobierno era una forma de potenciar en sus tareas cotidianas a actores sociales que realizan una actividad efectiva en problemáticas de salud pública y que cuentan a su vez -al menos en teoría- con un alto impacto dentro de los sectores populares. El reconocimiento estatal de grupos postergados es una forma indirecta de hacer política, de construir adhesiones, en tiempos electorales. Para el mundo evangélico el Programa representaba la posibilidad de establecer una relación duradera con el Estado, pasando de una etapa instrumental o meramente táctica con diferentes ministerios a un periodo más estable, visible, de consolidación dentro de las instituciones públicas y de disputa con la hegemonía católica en los dominios instituidos de la vida social.

En este juego de analogías entre las redes estatales y religiosas, el Programa RecuperarInclusión tiende a introducir en su método de trabajo elementos propios de las estrategias de tratamiento espiritual que desarrollan católicos y evangélicos (especialmente, la metodología de tratamiento que emplea dentro del catolicismo la Fundación Valdocco y el 
Programa Josué en el mundo evangélico). Por un lado, se prioriza el modelo ambulatorio sobre la internación. Las Casas Educativas Terapéuticas (CET), y los Centros Preventivos Locales de las Adicciones (CePLA) fueron ideados como puntos de encuentro, "casas que reciben", para un abordaje integral de las adicciones desde una perspectiva que incluye al deporte, al juego, la educación, el aprendizaje de oficios, la atención terapéutica y al entorno familiar en la recuperación de la persona. Por otro lado, la comprensión integral en el tratamiento de consumos problemáticos, introduce entre sus áreas de competencia, no sólo el desarrollo social, mental y físico de las personas sino también, el desarrollo espiritual que queda a cargo de una nueva figura: el "vitalizador". La meta de la vitalización es promover valores positivos de naturaleza religiosa en el acompañamiento tanto de las personas como del equipo profesional. Talleres, cursos, eventos artísticos, encuentros de contención y formación son algunas de las actividades que este nuevo actor social del Estado despliega en el territorio. Nuestro trabajo de campo nos permitió reconocer en el diseño y ejecución de las tareas del vitalizador las acciones coordinadas de movimientos eclesiales católicos -es el caso del Movimiento de la Palabra de Dios- y personalidades destacadas del medio evangélico, sobre todo referentes juveniles del ambiente bautista y pentecostal. La imagen del vitalizador, en un sentido abstracto, incorpora entonces la acción concreta de grupos cristianos que trasladan el punto de vista religioso a los modelos estatales de intervención en adicciones.

En resumen, la génesis social de los procesos de articulación entre las redes públicas y evangélicas nos permite reconocer ciertas analogías en lo que concierne a las formas de institucionalización que dinamizan al mundo de la política y al de la religión. En el encuentro circunstancial entre ambas realidades, los evangélicos se convierten en actores a través de los cuales el Estado ejecuta políticas de salud en consumos problemáticos, capitalizando su alcance territorial, su metodología y experiencia. Y, a su vez, las estructuras gubernamentales introducen -no sin tensiones y resistencias- el punto de vista espiritual en el desarrollo de políticas públicas, es decir, en los programas y planes de acción que le permiten al Estado definir prioridades y actuar sobre ellas.

\section{CONCLUSIONES}

Para concluir, podemos retomar el argumento central del artículo, relativo a los puntos de encuentro entre dos dinámicas emergentes de institucionalización. Una se origina en lo que denominamos impulsos de "espiritualización terapéutica" de las iglesias evangélicas, cuyo grado más alto de organización es el Programa Vida y los cursos de capacitación OSTE y, la otra, surge estratégicamente desde el Estado a través del Programa Recuperar Inclusión. Como intentamos mostrar a lo largo del artículo, ambos procesos reconocen rasgos análogos respecto de: a) las funciones alternativas del carisma y la burocracia en el diseño de planes estratégicos; b) la centralidad de los liderazgos secundarios; y c) la estructura reticular de sus instituciones, basada en la conexión operativa entre unidades interdependientes de trabajo.

Durante dos años (2014 y 2015), el Estado y las iglesias coincidieron en un plan de atención de las adicciones, en el que las políticas públicas lograron potenciar los dispositivos existentes en el mundo evangélico. El Programa Recuperar Inclusión representa una red macro de carácter público capaz de establecer articulaciones estables con las redes intermedias de grupos religiosos distintos al catolicismo. La novedad de esta política radica, sobre todo, en el carácter legítimo y protagónico que adoptaron los modelos evangélicos de tratamiento, abandonando así los circuitos informales de la vida pública para aparecer como referentes destacados, oficialmente reconocidos, en el abordaje de las adicciones.

La sociogénesis de los dispositivos evangélicos de rehabilitación de usuarios de drogas nos permite reconocer una faceta poco 
explorada de este grupo religioso y su relación con los debates públicos de salud. La cuestión de las adicciones habilita una estrategia de intervención sobre la juventud que no apunta genéricamente a las poblaciones internas de las iglesias o a un sector difuso de la sociedad, sino a un segmento específico e invisibilizado de los jóvenes que a causa de los consumos problemáticos ocupan posiciones liminares, de tránsito, entre diferentes grupos de pertenencia. Enfrentan, por ejemplo, ciclos inestables de cercanía y distancia con su familia nuclear, con la escuela, los espacios laborales o institutos de menores, con diversos entornos de amigos, con actividades culturales de intensa sociabilidad -es el caso recurrente del fútbol o la música- y también con ambientes de consumo, delincuencia o encierro, que a veces se oponen y otras coinciden con los contextos señalados.

En este horizonte intermitente de referencias, el templo evangélico suele ser un ámbito más, que contiene tanto como excluye o niega la cuestión de las adicciones y su tratamiento. Por eso, la capacitación en drogodependencia corresponde a ministerios específicos, muchas veces aislados, que conviven con el sentimiento de aceptación, desconfianza o desinterés de diversos sectores de la comunidad evangélica. Su expertise requiere del desarrollo de un conjunto de métodos y saberes que reelaboran contenidos de la biomedicina, el psicoanálisis, incluso, la autoayuda bajo el marco interpretativo de la perspectiva religiosa.

En términos sociológicos, podemos afirmar que su estrategia apunta a revertir las situaciones de liminalidad que coloca a los consumidores entre numerosos espacios de pertenencia y tránsito sostenido. Para ello ofrece un anclaje fuerte en un territorio de creencias, identificaciones, sociabilidades y circulaciones intensivas desde donde redefinir los principios generales de orden y relación con el mundo. Contrapone a la condición liminal que sitúa al sujeto "entre grupos", la pertenencia plena, estricta y cohesionada a las redes de confianza ${ }^{(67)}$ del Evangelio. Es esta lógica de intervención la que el Estado copia, perfecciona, replica a su propia velocidad y en sus propios términos.

\section{REFERENCIAS BIBLIOGRÁFICAS}

1. Marostica M. La iglesia evangélica en la Argentina como nuevo movimiento social. Sociedad y Religión. 1994;(12):3-21.

2. Carbonelli M, Mosqueira M. Luis Palau en Argentina: construcción mediática del campo evangélico, disputa por el espacio público y nuevas formas de territorialidad. Enfoques. $2008 ; 20(2): 65-87$

3. Carbonelli M, Mosqueira M. "Militantes del Señor": cosmología y praxis evangélica sobre el espacio público. Sociedad y Religión. 2010;20(3233):108-123.

4. Algranti JM. Política y religión en los márgenes: nuevas formas de participación social de las megaiglesias evangélicas en la Argentina. Buenos Aires: Ediciones Ciccus; 2010.
5. Carbonelli M. Pan y palabras; la inserción evangélica en la gestión pública en Argentina. Religião \& Sociedade. 2015;35(2):73-95.

6. Mosqueira M. Redimir a política: experiências de militância de jovens evangélicos da Argentina. Desidades. 2015;(8):9-18.

7. Semán P. Bajo continuo; exploraciones descentradas sobre cultura popular y masiva. Buenos Aires: Gorla; 2006.

8. Semán P, Battaglia A. De la industria cultural a la religión: nuevas formas y caminos para el sacerdocio. Civitas. 2012;12(3):439-452.

9. Algranti J. La industria del creer: sociología de las mercancías religiosas. Buenos Aires: Biblos; 2013.

10. Lago L. El cuerpo como territorio de creencias: un análisis sobre la relación entre corporalidad 
juvenil y música cristiana evangélica. Questión. 2016;1(52):42-56.

11. Mosqueira M. La manifestación de los hijos de Dios: reconfiguración del campo evangélico y emergencia del sujeto juvenil cristiano en la Argentina (1960-2000). Revista de Ciencias SocialesSegunda Época. 2016;(30):53-83.

12. Mosqueira M. "Hasta lo último de la tierra": consolidación y transnacionalización del rock cristiano argentino. Journal of the Sociology and Theory of Religion. 2016;5(1):77-101.

13. Brardinelli R. De iglesias y pabellones inventados: paradigmas carcelarios y "conversiones religiosas". Revista de Ciencias Sociales-Segunda Época. 2012;(22):7-26.

14. Brardinelli R, Algranti J. La reinvención religiosa del encierro: hermanitos, refugiados y cachivaches en los penales bonaerenses. Buenos Aires: Centro Cultural de la Cooperación, Universidad Nacional de Quilmes; 2013.

15. Manchado M. Reciprocidades y gubernamentalidad tras la inserción del dispositivo religioso en cárceles de mediana y máxima seguridad de la provincia de Santa Fe (Argentina). Revista de Antropología Social. 2016;25(1):35-60.

16. Vallejos A. Unidad 25, la cárcel-iglesia; origen, auge y transformación de la primera cárcel destinada a presos de confesión cristiana evangélica pentecostal. [Tesis de maestría]. Buenos Aires: Universidad Nacional de la Matanza; 2016.

17. Míguez D. Jóvenes en riesgo y conversión religiosa; esquemas cognitivos y transformación de la identidad en iglesias pentecostales e instituciones de minoridad. Sociedad y religión. 2000;(2021):1-23.

18. Míguez D. Los universos morales en el mundo del delito, las lógicas de la reconversión en contextos de institucionalización. Revista de Ciencias Sociales-Segunda Época. 2012;(22):45-63.

19. Míguez D. Inscripta en la piel y en el alma: cuerpo e identidad en profesionales, pentecostales y jóvenes delincuentes. Religião e Sociedade. $2002 ; 22(1): 21-56$.

20. Míguez D. Identidades conflictivas: droga, delito y religión en un programa de rehabilitación de adictos. Revista Cultura y Religión. 2007;1(1):88-107.

21. Epele M. Sujetar por la herida; una etnografía sobre drogas, pobreza y salud. Buenos Aires: Paidós; 2010.
22. Castilla MV, Lorenzo G. Consumo de pasta base/paco, prácticas de rescate y religiosidad pentecostal. Sociedad y religión. 2013;23(39):54-78.

23. Jones D, Cunial S. Más allá de los límites del Estado; instituciones católicas y evangélicas de partidos del Gran Buenos Aires (Argentina) en la implementación de políticas públicas sobre drogas. Desafíos. 2017;29(2):85-123.

24. Güelman M. Encontrar el sentido de la vida: rehabilitación y conversión en dos comunidades terapéuticas religiosas de redes internacionales. [Tesis de maestría]. Buenos Aires: Universidad Nacional General Sarmiento; 2017.

25. Sanchez ZM, Nappo SA. A religiosidade, a espiritualidade e o consumo de drogas. Archives of Clinical Psychiatry. 2007;34(S1):73-81.

26. Geppert C, Bogenschutz MP, Miller WR. Development of a bibliography on religion, spirituality and addictions. Drug and Alcohol Review. 2007;26(4):389-395.

27. Koenig $\mathrm{H}$, King $\mathrm{D}$, Carson VB. Handbook of religion and health. 2a ed. London: Oxford University Press; 2012.

28. Cantón Delgado M. Gitanos pentecostales: una mirada antropológica a la Iglesia Filadelfia en Andalucía. Sevilla: Signatura Demos; 2004.

29. Comas Arnau D. Un lugar para otra vida: los centros residenciales y terapéuticos del movimiento carismático y pentecostal en España. Madrid: Fundación Atenea Grupo GID; 2010.

30. Apud I, Romaní O. Medicine, religion and ayahuasca in Cataloni: considering ayahuasca networks from a medical anthropology perspective. International Journal of Drug Policy. 2017;39:28-36.

31. Castrillón Valderruten MC. Entre "teoterapias" y "laicoterapias": comunidades terapéuticas en Colombia y modelos de sujetos sociales. Psicologia \& Sociedade. 2008;20(1):80-90.

32. Galaviz G, Ortiz O. Estado laico y alternativas terapéuticas religiosas: el caso de México en el tratamiento de adicciones. Debates do NER. 2014;2(26):253-276.

33. Hernández OLO, Odgers Ortiz O. Renacer en Cristo: cuerpo y subjetivación en la experiencia de rehabilitación de adicciones en los centros evangélico pentecostales. Ciências Sociais e Religião. 2015;17(22):90-119.

34. Galaviz Granados G. Mujeres, adicción y rehabilitación: reflexiones desde la frontera noroeste 
de México. Salud Colectiva. 2015;11(3):367-379.

35. Mariz CL. "Embriagados no Espírito Santo": reflexões sobre a experiência pentecostal e o alcoolismo. Antropolítica. 2003;(15):61-82.

36. Sánchez ZM, Nappo SA. Intervenção religiosa na recuperação de dependentes de drogas. Revista de Saúde Pública. 2008;42(2):265-272.

37. Rocha MLA, Guimarães MBL, Cunha MB. O processo de recuperação do uso indevido de drogas em igrejas pentecostais Assembléia de Deus. Interface - Comunicação, Saúde, Educação. 2012;16(40):177-190.

38. Ribeiro FML, Minayo MCS. As comunidades terapêuticas religiosas na recuperação de dependentes de drogas: o caso de Manguinhos, RJ, Brasil. Interface - Comunicação, Saúde, Educação. 2015;19(54):515-526.

39. Saizar M. Reflexiones en torno de la complementariedad terapéutica entre usuarios del yoga en el Área Metropolitana de Buenos Aires (Argentina). Mitológicas. 2006;21:23-46.

40. Carbonelli M, Irrazábal G. Católicos y evangélicos ¿alianzas religiosas en el campo de la bioética argentina? Nómadas-Critical Journal of Social and Juridical Sciences. 2010;26(2):269-284.

41. Bordes M. Entre el arte de curar y la profesionalización; aportes para el estudio de la práctica médica alternativa o nueva era a partir de las trayectorias socio-ocupacionales de especialistas. Revista de Antropología Experimental. 2009;(9):55-73.

42. Molina AI. Enfermedad, terapia y las expresiones de lo sagrado; una síntesis sobre medicinas y religiosidades en Argentina. Ciências Sociais e Religião. 2015;17(22):15-37.

43. Puglisi R. La "energía" que crea y sana: representaciones corporales y prácticas terapéuticas en devotos de Sai Baba. Ciências Sociais e Religião. 2015;17(22):71-89.

44. Irrazábal G. La religión en las decisiones sobre aborto no punible en la Argentina. Revista Estudos Feministas. 2015;23(3):735-759.

45. Irrazábal G. Religión y salud: la intervención pública de agentes religiosos católicos formados en bioética en el debate parlamentario sobre la muerte digna en la Argentina. Salud Colectiva. 2015;11(3):331-349.

46. Toniol R. Espiritualidade que faz bem: pesquisas, políticas públicas e práticas clínicas pela promoção da espiritualidade como saúde. Sociedad y Religión. 2015;25(43):110-146.

47. Olmos Álvarez AL. "Venid a mí todos los afligidos": salud, enfermedad y rituales de sanación en el movimiento católico carismático del Padre Ignacio. Ciências Sociais e Religião. 2015; 17(22):52-70.

48. Giménez Béliveau V. Terapéuticas católicas, males modernos: procesos de sanación y exorcismo en la Argentina. Sociedad y Religión. 2017;27(47):33-59.

49. Ramírez Hita S. Donde el viento llega cansado: sistemas y prácticas de salud en la ciudad de Potosí. La Paz: Cooperación Italiana; 2005.

50. Ramírez Hita S. Entre calles estrechas; gitanos: prácticas y saberes médicos. Barcelona: Bellaterra; 2007.

51. De leso LC. Espiritualidad y poder superior en el tratamiento de adicciones con jóvenes; sistematización de una experiencia en una comunidad terapéutica. En: Grupo de Estudios en Juventudes, Facultad de Trabajo Social, Universidad Nacional de La Plata. Estudios sobre juventudes en Argentina II. Salta: Red de Investigadoras/es en Juventudes de Argentina, Editorial de la Universidad Nacional de Salta; 2012. p. 216-232.

52. Gutiérrez Portillo AA. Purificando almas: alcohólicos anónimos en Bacalar, Carlos A. Madrazo y Ramonal, Quintana Roo. [Tesis de doctorado]. México DF: Universidad Nacional Autónoma de México; 2014.

53. Manzano V. Cultura, política y el "problema de las drogas" en la Argentina, 1960-1980. Apuntes de Investigación del CECYP. 2014;24(1):51-78.

54. Manzano V. "Y, ahora, entre gente de clase media como uno": culturas juveniles, drogas y política en la Argentina, 1960-1980. Contemporánea. 2014;5(5):85-104.

55. Federico M, Ramirez I. Historia de la droga en la Argentina. Buenos Aires: Aguilar; 2015.

56. Levin LG. Las adicciones como construcción social: conocimientos, posicionamiento público, e implementación estatal de tratamientos. [Tesis de doctorado]. Buenos Aires: Universidad Nacional de Quilmes; 2016.

57. Garbi SL. De aislamientos y encierros: modos "legos" y "expertos" de tratar los consumos problemáticos de drogas en el Área Metropolitana de Buenos Aires. [Tesis de doctorado]. Buenos Aires: Universidad de Buenos Aires. 2016. 
58. Galante A, Rossi D, Pawlowicz MP, Ralón G. Del adicto recuperado al operador socioterapéutico: la importancia de la intervención estatal en los procesos de profesionalización [Internet]. Buenos Aires: X Jornadas de Sociología; 2013 [citado 1 jul 2017]. Disponible en: https://tinyurl. com/ycdkhka7.

59. Novelli C, Mann P. Guía para educadores y líderes sobre "la verdadera historia de Mary Juana" o cómo te daña la marihuana. Buenos Aires: Programa Andrés; 1989.

60. Su púlpito está en los boliches. El Puente. 1987;2(27):24.

61. Spadafora AM. Religión, política y estética: el pentecostalismo en la Argentina de los '90. [Tesis de doctorado]. Buenos Aires: Universidad de Buenos Aires; 2004.

62. Camarotti AC, Di Leo PF. Cuerpos juveniles y consumos de drogas: entre la negación y la recreación. Ciencias Sociales. 2007;(67):34-35.
63. Camarotti AC, Kornblit AL. Abordaje integral comunitario de los consumos problemáticos de drogas: construyendo un modelo. Salud Colectiva. 2015;11(2):211-221.

64. Aureano GR. La construction politique du toxicomane dans I'Argentine postautoritaire; un cas de citoyenneté à basse intensité. [Thèse de Philosphiæ Doctor]. Montreal: Université de Montréal; 1997.

65. Mano a mano con Boanerges: se pasaron las puertas del templo. El Puente. 1998;12(152):31.

66. Elias N. Sociología fundamental. Barcelona: Gedisa; 2006.

67. Tilly C. Confianza y gobierno. Buenos Aires: Amorrortu; 2010.

\section{FORMA DE CITAR}

Algranti J, Mosqueira M. Sociogénesis de los dispositivos evangélicos de "rehabilitación" de usuarios de drogas en Argentina. Salud Colectiva. 2018;14(2):305-322. doi: 10.18294/sc.2018.1521.

Recibido: 10 de julio de 2017 | Versión final: 2 de diciembre de 2017 | Aprobado: 28 de diciembre de 2017

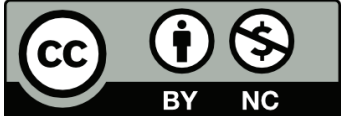

Este obra está bajo una licencia de Creative Commons Reconocimiento-NoComercial 4.0 Internacional. Reconocimiento - Permite copiar, distribuir y comunicar públicamente la obra. A cambio, se debe reconocer y citar al autor original. No Comercial - Esta obra no puede ser utilizada con finalidades comerciales, a menos que se obtenga el permiso. 